\title{
The Use of Tranexamic Acid for Upper Gastrointestinal Bleeding by Medical and Surgical Intensivists: A Single Center Experience
}

\author{
Jason Chertoff ${ }^{\mathrm{a}, \mathrm{c}}$, Grant Lowther ${ }^{\mathrm{b}}$, Hassan Alnuaimat ${ }^{\mathrm{a}}$, Ali Ataya ${ }^{\mathrm{a}}$
}

\begin{abstract}
Background: Tranexamic acid (TXA) may be beneficial in the management of upper gastrointestinal bleeding (UGIB). We sought to investigate how frequently intensivists at our academic institution use TXA for patients with UGIB, and to investigate whether the utilization rate of TXA differs between surgical and medical intensivists, and provide an updated literature review on the subject.
\end{abstract}

Methods: We performed a retrospective cohort study of patients admitted for UGIB to the surgical intensive care unit (SICU) and the medical intensive care unit (MICU) at our academic healthcare facility (University of Florida Health - Shands Hospital) from January 1, 2013 to December 31, 2016. The patients were categorized as receiving or not receiving TXA. The overall utilization rate of TXA was calculated, and the utilization rates for the MICU and SICU were compared using a twosample test for equality of two proportions with continuity correction.

Results: The study cohort included a total of 1,829 patients with a diagnosis of UGIB. Of those, 988 were treated in the MICU and 841 were treated in the SICU. Of the 988 patients in the MICU, six received TXA $(0.61 \%)$, while $10(1.19 \%)$ of the 841 patients in the SICU received TXA. The overall utilization rate of TXA was $0.87 \%$. The odds of receiving TXA in the SICU were 1.97 times greater than in the MICU (odds ratio (OR): 1.97, 95\% confidence interval (CI): $0.74-5.2, \mathrm{P}=1.83)$.

Conclusions: Our study suggests that TXA may be underused in the management of UGIB, and that the utilization rate does not differ significantly between surgical and medical intensivists.

Keywords: Tranexamic acid; Upper gastrointestinal bleeding; Intensivists

Manuscript submitted July 24, 2017, accepted August 8, 2017

aDivision of Pulmonary, Critical Care, and Sleep Medicine, University of Florida College of Medicine, Gainesville, FL, USA

bepartment of Internal Medicine, University of Florida College of Medicine, Gainesville, FL, USA

${ }^{\mathrm{c} C}$ Corresponding Author: Jason Chertoff, University of Florida College of Medicine, 1600 SW Archer Road, M452, PO Box 100225, Gainesville, FL 32610, USA. Email: jason.chertoff@medicine.ufl.edu

doi: https://doi.org/10.14740/gr891w
Introduction

Tranexamic acid (TXA) is an antifibrinolytic agent with a well-established role in the management of hemorrhagic shock due to trauma, reducing transfusion requirements in surgeries necessitating cardiopulmonary bypass, orthopedic surgeries, and abnormal uterine bleeding [1-6]. The CRASH-2 trial demonstrated the utility of TXA in trauma surgery where TXA reduced all-cause mortality and death due to blood loss [1]. Moreover, TXA decreased menstrual blood loss due to abnormal uterine bleeding by $45 \%$ over the course of two menstrual cycles [2], while the WOMAN trial demonstrated that TXA reduced death due to rebleeding in patients experiencing postpartum hemorrhage $[2,6]$. These indications are widely supported by evidence and when used for these applications, TXA has a favorable risk/benefit profile and low cost $[4,7,8]$.

Recent evidence suggests TXA likely has a role in the management of upper gastrointestinal bleeding (UGIB) since it often reduces acute transfusion requirements, rebleeding, and the need for surgical intervention without increasing the risks for thrombotic events [4, 9-13].

The mortality from UGIB remains high despite improvements in endoscopic therapy, underscoring the need for new and/or adjunctive therapies such as TXA. Specifically, mortality is most closely associated with rebleeding in UGIB, a complication in which TXA shows promise [14]. Most data surround TXA in UGIB due to peptic ulcer disease, but there have been reports of TXA being used for the management of UGIB from other causes as well [15].

Unfortunately, TXA is not yet considered to be standard of care in the same fashion as anti-ulcer/endoscopic therapy due to inadequate evidence in the specific context of UGIB [16, 17]. Of the meta-analyses completed on this topic, a definitive conclusion could not be reached on the usefulness of TXA in UGIB, largely due to the paucity of high quality studies [16, 17]; ongoing randomized, double-blind trials are further investigating the topic, specifically investigating TXA's effect on morbidity, mortality, transfusion requirements and surgical intervention (HALT-IT, NCT01658124) [18]. Despite this, the utilization rate of TXA in UGIB is roughly $1 \%$ [19].

Although TXA is not considered standard of care, it still may have a role in the management of UGIB. The aim of our study was to examine the current rate of TXA utilization in patients with UGIB. Moreover, due to TXA's more established 
Table 1. Rates of TXA Use in UGIB in the SICU and MICU

\begin{tabular}{llllll} 
& Receiving TXA & Not receiving TXA & Total patients & TXA utilization (\%) & P value \\
\hline MICU population & 6 & 982 & 988 & 0.61 & 0.183 \\
SICU population & 10 & 831 & 841 & 1.19 \\
Total population & 16 & 1,813 & 1,829 & 0.87 \\
\hline
\end{tabular}

role in the trauma and surgical settings, we hypothesize that TXA is more frequently used by surgical intensivists than medical intensivists.

\section{Methods}

We performed a retrospective cohort study of patients over the age of 18 admitted for UGIB to either the surgical intensive care unit (SICU) or medical intensive care unit (MICU) at the University of Florida Health - Shands Hospital from January 1, 2013 to December 31, 2016. The patients were categorized as receiving or not receiving TXA. The overall utilization rate of TXA was calculated, and the utilization rates for the MICU and SICU were compared using a two-sample test for equality of two proportions with continuity correction. This study was conducted under the approval of the responsible institutional review board (IRB).

\section{Results}

During the aforementioned dates, the study cohort included a total of 1,829 patients admitted to an ICU with a diagnosis of UGIB (Table 1). Of those, 988 were admitted to the MICU and 841 were admitted to the SICU. Of those admitted to the MICU, six $(0.61 \%)$ received TXA and of those admitted to the SICU, $10(1.19 \%)$ received TXA. There was a 1.96 times greater chance a patient in the SICU received TXA than a patient in the MICU, but this was not statistically significant (risk ratio (RR): $1.96,95 \%$ confidence interval $(\mathrm{CI}): 0.715-5.365$, $\mathrm{P}=0.183)$. Similarly, the risk difference of $0.0058(95 \% \mathrm{CI}$ : $-0.003-0.014)$ was not significantly significant $(\mathrm{P}=0.183)$.

\section{Discussion}

TXA has an established role in the management of bleeding across a disparate set of disciplines [1-6]. The evidence for its use in dysfunctional uterine bleeding, cardiopulmonary bypass surgery, traumatic bleeding, and orthopedic surgery is strong, and is considered the standard of care in these settings. CRASH-2 established TXA as part of the standard of care in reducing all-cause mortality and death due to bleeding in adult trauma patients. Additionally, Matteson et al and Lukes et al established that TXA was an effective pharmacological agent for the management of abnormal uterine bleeding. Specifically, TXA demonstrated a reduction in average menstrual bleeding of $69.6 \mathrm{~mL}$, or a reduction of $40.4 \%$.

In surgeries where cardiopulmonary bypass is necessary,
TXA has a well-established role. Within the cardiopulmonary bypass circuit, both coagulation and fibrinolysis are activated, which predisposes patients to postoperative bleeding, something that TXA has been shown to mitigate by reducing postoperative transfusion requirements [4, 20]. Finally, orthopedic surgeries frequently require a tourniquet to create a dry surgical field, which is associated with local increases in fibrinolysis and post-operative bleeding [21]; TXA has been shown to reduce postoperative blood loss under these circumstances due to its antifibrinolytic properties [4].

TXA is an attractive option in the management of bleeding, not only due to its hemostatic properties, but also because of its safety profile. Specifically, TXA does not increase the risk of thrombotic events despite inhibiting thrombolysis, making it a safe and effective therapy [4, 6-8].

In the setting of UGIB, the utility of TXA remains unclear despite substantial evidence that it reduces the overall transfusion requirements and decreases the risk surgical intervention [4, 9-13]. In a meta-analysis of seven studies, Jiang et al showed that TXA reduced the need for surgical intervention and the rate of bleeding in UGIB. These results lend veracity to Sabovic et al, whose pilot study demonstrated that TXA reduced rebleeding and repeat endoscopic intervention; other randomized control trials have shown similar results $[11,12]$.

Our retrospective review of patients admitted to either the SICU or MICU for UGIB shows the utilization rate of TXA for UGIB to be $0.87 \%$, which is similar to the $1 \%$ that has been documented in prior studies [19]. Furthermore, our data support that the utilization rate of TXA for UGIB does not differ between medical and surgical intensivists. To our knowledge, this is the first study that analyzed and compared the utilization rate of TXA among intensivists across disciplines.

There are enough data to support a beneficial role of TXA in UGIB management. At only $0.87 \%$, which is a rate seen in prior studies, our hypothesis that TXA in UGIB is underutilized was confirmed. This underutilization is likely due to a lack of consensus guidelines recommending TXA for this indication and the lack of robust data demonstrating definitive efficacy. However, given the plethora of positive trials and reports, in conjunction with the safety and low cost of TXA, more frequent use of TXA in UGIB would be appropriate. The standard of care continues to be anti-ulcer and endoscopic therapy, but if this fails, TXA should be considered as an adjunct. Despite it not being in any consensus guidelines, we feel TXA should not be outright excluded from a physician's clinical arsenal, especially considering its agreeable risk/benefit profile.

One might postulate a higher utilization rate of TXA by surgical intensivists than medical intensivists for UGIB. Our data do not confirm this; however, it shows a trend of higher utilization rate of TXA in patients with UGIB treated by surgi- 
cal intensivists. It is unclear why this is the case, but it may be due to a lack of knowledge among surgical and medical intensivists about the growing evidence supporting the use of TXA in UGIB. Of course it could also be due to the study's lack of power to find a statistical difference, because of the overall low utilization of TXA in the study population.

Our study has limitations worth mentioning. First, due to IRB constraints, we were not able to collect certain demographic information that could have potentially confounded our results. Second, this was a single institute retrospective study, which limits generalizability since our institution may have radically different TXA utilization rates than others in the US. Third, due to type of data collected we were not able to account for any preexisting medical comorbidities or illness severity (i.e., SOFA, APACHE, etc.), which may have significantly biased our results. Finally, although our data showed no difference in utilization rates between the SICU and MICU, this could be a consequence of our study being underpowered.

\section{Conclusion}

TXA has a well-established role in improving hemostasis across many disciplines. The evidence supporting it use in UGIB, although not as robust, suggests a potential benefit. Few studies have investigated how frequently TXA is used in UGIB, and whether its use differs between surgical and medical intensivists. Our study shows that there is a low utilization rate of TXA in the management of UGIB, and that this phenomenon is present in both surgical and medical disciplines. However, depending on the results of the HALT-IT trial, TXA may become more frequently used.

\section{Author Contributions}

Conception and design: JC, GL, and AA. Analysis and interpretation: JC, GL, and AA. Drafting the manuscript for important intellectual content: JC, GL, and AA.

\section{References}

1. Williams-Johnson JA, McDonald AH, Strachan GG, Williams EW. Effects of tranexamic acid on death, vascular occlusive events, and blood transfusion in trauma patients with significant haemorrhage (CRASH-2) A randomised, placebo-controlled trial. West Indian Med J. 2010;59(6):612-624.

2. Matteson KA, Rahn DD, Wheeler TL, 2nd, Casiano E, Siddiqui NY, Harvie HS, Mamik MM, et al. Nonsurgical management of heavy menstrual bleeding: a systematic review. Obstet Gynecol. 2013;121(3):632-643.

3. Lukes AS, Freeman EW, Van Drie D, Baker J, Adomako TL. Safety of tranexamic acid in women with heavy menstrual bleeding: an open-label extension study. Womens Health (Lond). 2011;7(5):591-598.

4. Dunn CJ, Goa KL. Tranexamic acid: a review of its use in surgery and other indications. Drugs. 1999;57(6):10051032.

5. Ker K, Edwards P, Perel P, Shakur H, Roberts I. Effect of tranexamic acid on surgical bleeding: systematic review and cumulative meta-analysis. BMJ. 2012;344:e3054.

6. Collaborators WT. Effect of early tranexamic acid administration on mortality, hysterectomy, and other morbidities in women with post-partum haemorrhage (WOMAN): an international, randomised, double-blind, placebo-controlled trial. Lancet. 2017;389(10084):2105-2116.

7. McCormack PL. Tranexamic acid: a review of its use in the treatment of hyperfibrinolysis. Drugs. 2012;72(5):585617.

8. Tengborn L, Blomback M, Berntorp E. Tranexamic acid - an old drug still going strong and making a revival. Thromb Res. 2015;135(2):231-242.

9. Jiang M, Chen P, Gao Q. Systematic review and net-work meta-analysis of upper gastrointestinal hemorrhage interventions. Cell Physiol Biochem. 2016;39(6):2477-2491.

10. Sabovic M, Lavre J, Vujkovac B. Tranexamic acid is beneficial as adjunctive therapy in treating major upper gastrointestinal bleeding in dialysis patients. Nephrol Dial Transplant. 2003;18(7):1388-1391.

11. von Holstein CC, Eriksson SB, Kallen R. Tranexamic acid as an aid to reducing blood transfusion requirements in gastric and duodenal bleeding. Br Med J (Clin Res Ed). 1987;294(6563):7-10.

12. Biggs JC, Hugh TB, Dodds AJ. Tranexamic acid and upper gastrointestinal haemorrhage - a double-blind trial. Gut. 1976;17(9):729-734.

13. Flores S, Aviles C, Rada G. Is tranexamic acid effective for acute upper gastrointestinal bleeding? Medwave. 2015;15(Suppl 3):e6330.

14. Katschinski B, Logan R, Davies J, Faulkner G, Pearson J, Langman M. Prognostic factors in upper gastrointestinal bleeding. Dig Dis Sci. 1994;39(4):706-712.

15. McCormick PA, Ooi H, Crosbie O. Tranexamic acid for severe bleeding gastric antral vascular ectasia in cirrhosis. Gut. 1998;42(5):750-752.

16. Gluud LL, Klingenberg SL, Langholz SE. Systematic review: tranexamic acid for upper gastrointestinal bleeding. Aliment Pharmacol Ther. 2008;27(9):752-758.

17. Bennett C, Klingenberg SL, Langholz E, Gluud LL. Tranexamic acid for upper gastrointestinal bleeding. Cochrane Database Syst Rev. 2014;11:CD006640.

18. Roberts I, Coats T, Edwards P, Gilmore I, Jairath V, Ker $\mathrm{K}$, Manno D, et al. HALT-IT - tranexamic acid for the treatment of gastrointestinal bleeding: study protocol for a randomised controlled trial. Trials. 2014;15:450.

19. Manno D, Ker K, Roberts I. How effective is tranexamic acid for acute gastrointestinal bleeding? BMJ. 2014;348:g1421.

20. Guenther CR. Pro: tranexamic acid is better than aprotinin in decreasing bleeding after cardiac surgery. J Cardiothorac Vasc Anesth. 1994;8(4):471-473.

21. Klenerman L, Chakrabarti R, Mackie I, Brozovic M, Stirling Y. Changes in haemostatic system after application of a tourniquet. Lancet. 1977;1(8019):970-972. 